\title{
Biocontrol of Potato Common Scab is Associated with High Pseudomonas fluorescens LBUM223 Populations and Phenazine-1-Carboxylic Acid Biosynthetic Transcript Accumulation in the Potato Geocaulosphere
}

\author{
Tanya Arseneault, Claudia Goyer, and Martin Filion
}

First and third authors: Université de Moncton, Department of Biology, Moncton, NB, Canada; first author: University of Reading, School of Biological Sciences, Reading, UK; and second author: Potato Research Center, Agriculture and Agri-Food Canada, Fredericton, NB, Canada. Accepted for publication 12 April 2016.

\begin{abstract}
Arseneault, T., Goyer, C. and Filion, M. 2016. Biocontrol of potato common scab is associated with high Pseudomonas fluorescens LBUM223 populations and phenazine-1-carboxylic acid biosynthetic transcript accumulation in the potato geocaulosphere. Phytopathology 106:963-970.

Pseudomonads are often used as biocontrol agents because they display a broad range of mechanisms to control diseases. Common scab of potato, caused by Streptomyces scabies, was previously reported to be controlled by Pseudomonas fluorescens LBUM223 through phenazine-1-carboxylic acid (PCA) production. In this study, we aimed at characterizing the population dynamics of LBUM223 and the expression of $p h z C$, a key gene involved in the biosynthesis of PCA, in the rhizosphere and geocaulosphere of potato

plants grown under controlled and field conditions. Results obtained from controlled experiments showed that soil populations of LBUM223 significantly declined over a 15 -week period. However, at week 15, the presence of $S$. scabies in the geocaulosphere was associated with significantly higher populations of LBUM223 than when the pathogen was absent. It also led to the detection of significantly higher $p h z C$ gene transcript numbers. Under field conditions, soil populations of LBUM223 followed a similar decline in time when a single inoculation was applied in spring but remained stable when reinoculated biweekly, which also led to greater $p h z C$ gene transcripts accumulation. Taken together, our findings suggest that LBUM223 must colonize the potato geocaulosphere at high levels $\left(10^{7} \mathrm{bacteria} / \mathrm{g}\right.$ of soil) in order to achieve biocontrol of common scab through increased PCA production.
\end{abstract}

Pseudomonads are well known for their biological control capacity against several plant pathogens (Haas and Défago 2005; Weller 2007). They show great potential to be commercialized as biocontrol agents because they are easy to grow, can easily colonize the rhizosphere surrounding the roots of numerous plant species, adapt to a wide variety of environmental conditions, and produce numerous bioactive metabolites (Chin-A-Woeng et al. 2003; Weller 2007). It has been demonstrated that disease-suppressive soils are often rich in antibiotic-producing Pseudomonas spp. (Mazurier et al. 2009; Weller et al. 2002). One of the main biocontrol mechanism used by pseudomonads is antibiosis, which reduces disease symptoms through the production of antimicrobial compounds, including phenazines (Raaijmakers et al. 2002). Phenazines exist in several chemical forms, including hydroxyphenazine, pyocyanine, and phenazine-1-carboxylic acid (PCA), which differ in their properties, but all of which possess redox activity (Price-Whelan et al. 2006). Phenazines may also serve as cell signals, and can contribute to biofilm formation and bacterial survival, among many functions (Pierson and Pierson 2010).

We have previously isolated a Pseudomonas fluorescens strain (LBUM223) that is capable, under in vitro conditions, of inhibiting the growth of Streptomyces scabies (St-Onge et al. 2011), the causal agent of common scab of potato (Lambert and Loria 1989). Subsequent experiments have revealed that LBUM223 was also able to control common scab symptoms in planta under controlled (Arseneault et al. 2013) and field (Arseneault et al. 2015) conditions. The development

Corresponding author: M. Filion; E-mail address: martin.filion@umoncton.ca

*The $e$-Xtra logo stands for "electronic extra" and indicates that three supplementary figures are published online.

http://dx.doi.org/10.1094/PHYTO-01-16-0019-R

(C) 2016 The American Phytopathological Society and use of an isogenic mutant incapable of producing PCA (St-Onge et al. 2011) has allowed the demonstration that the biocontrol capability of LBUM223 was closely linked to its PCA-producing ability, because only the wild-type strain was able to reduce common scab symptoms (Arseneault et al. 2013). PCA production by several other strains of Pseudomonas spp. has also been proven essential in the biocontrol of many other diseases, including takeall of wheat (Thomashow et al. 1990; Yang et al. 2011), root rot of wheat (Mavrodi et al. 2012) and bean (D'aes et al. 2011), cypress canker (Raio et al. 2011), and damping-off of pea (Timms-Wilson et al. 2000).

The biosynthesis of PCA has been associated with a sevengene operon, phzABCDEFG (Mavrodi et al. 1998). Some of these genes, including $p h z C, p h z D, p h z E$, and $p h z F$, are essential for PCA production. The $p h z C$ gene, which encodes a type II 3-deoxyD-arabinoheptulosonate-7-phosphate synthase enzyme, is of particular interest because it is thought to be the first step of the biosynthetic pathway, diverting the metabolism of carbon into producing chorismic acid, a precursor of PCA (Mavrodi et al. 1998; Pierson and Pierson 2010). The production of PCA by $P$. fluorescens $2-79$, one the most studied Pseudomonas strains, was shown to be affected by several environmental factors, including $\mathrm{pH}$, temperature, and glucose source (Slininger and Shea-Wilbur 1995), as well as the presence of growth factors or minerals (Slininger and Jackson 1992). Moreover, in recent studies by Mavrodi and colleagues (2012, 2013), the population dynamics and PCA production of saprophytic Pseudomonas spp. in natural field soil was characterized, showing that PCA production is positively correlated with soil populations of Pseudomonas spp. and is increased in arid fields, being negatively affected by irrigation. The persistence of PCA in soil has recently been evaluated by treating the roots of potted plants with purified PCA. It was found that the half-life of PCA under these conditions was of 3.4 days, suggesting that a sustained production of the antibiotic (for example by maintaining high population levels of PCA-producing Pseudomonas spp.) is 
required to prevent rapid PCA degradation and maintain its biological effect (Mavrodi et al. 2013).

The soil population dynamics of $P$. fluorescens LBUM223 as well as its PCA biosynthetic operon expression in the rhizosphere and geocaulosphere (soil in close contact with potato tubers) is not yet characterized. A better understanding of the interaction occurring between S. scabies and LBUM223 in soil would help promote its use as a biocontrol agent against common scab of potato. Because the ability to produce PCA was previously demonstrated as being essential to disease control, the effect of different conditions on its biosynthetic transcriptional regulation by LBUM223 will give further insights into how it can contribute to the biocontrol observed. Therefore, the aims of this study were to (i) characterize the rhizosphere and geocaulosphere soil population dynamics of $P$. fluorescens LBUM223; (ii) characterize some physiological traits of LBUM223 potentially involved in rhizosphere and geocaulosphere soil colonization and survival; (iii) measure the transcriptional activity of $p h z C$, a key biosynthetic gene leading to PCA production in the rhizosphere and geocaulosphere; and (iv) determine whether a relationship exists between the previously observed biocontrol of common scab (Arseneault et al. 2015) and LBUM223's soil population dynamics or its expression of $p h z C$ under controlled and field conditions.

\section{MATERIALS AND METHODS}

P. fluorescens LBUM223 inoculum. Liquid inocula of wildtype LBUM223 and its isogenic phzC-mutant not producing PCA (St-Onge et al. 2011) were prepared in tryptic soy broth (TSB) (BD, Franklin Lakes, NJ) and grown to late log phase with shaking at $150 \mathrm{rpm}$ at $25^{\circ} \mathrm{C}$. The bacterial concentrations were estimated using spectrophotometer readings $(\lambda=600 \mathrm{~nm})$ and standard curves, as previously described (Arseneault et al. 2013). The genome of LBUM223 (GenBank accession number CP011117) (http://www. ncbi.nlm.nih.gov/genbank/), which was isolated from the rhizosphere of strawberry in a field in Bouctouche, Canada, has been sequenced (Roquigny et al. 2015).

Growth-chamber experiments. Experiments were performed as previously described (Arseneault et al. 2013). In summary, gleyed podzolic gray luvisol soil ( $\mathrm{pH} 6.7$ ), consisting of $62 \%$ sand, $25 \%$ silt, $13 \%$ clay, and $2.6 \%$ organic matter, was collected from experimental plots located at the Agriculture and Agri-Food Canada S. H. J. Michaud Research Farm (Bouctouche, NB, Canada) and sieved. Soil was mixed $3: 1$ with sand as a potting substrate and autoclaved $\left(121^{\circ} \mathrm{C}\right.$ for $60 \mathrm{~min}$ ) twice ( $48 \mathrm{~h}$ apart). The experimental set-up consisted of six treatments: (i) no inoculum (control), (ii) LBUM223, (iii) LBUM223 phzC-, (iv) S. scabies, (v) S. scabies + LBUM223, and (vi) S. scabies + LBUM223 phzC-, with nine replicates of each. Treatments containing LBUM223 or LBUM223 phzC- were inoculated once when seed tubers ('Kennebec') were planted using $20 \mathrm{ml}$ of inoculum at a concentration of $2.5 \times 10^{9} \mathrm{CFU} / \mathrm{ml}$. A soil-based inoculum of S. scabies (strain LBUM848) (St-Onge et al. 2008) was prepared as previously described by inoculating with a liquid culture (Arseneault et al. 2013), and mixed with the potting substrate at a concentration of approximately $5 \times 10^{7} \mathrm{CFU} / \mathrm{g}$ of soil prior to planting. Control plants received the same volume of water. Destructive harvesting of three replicates per treatment occurred at 5 , 10, and 15 weeks. The entire experiment was repeated a second time. Rhizosphere and geocaulosphere soils were sampled by wiping the first few millimeters of adhering soil from the roots and tubers, respectively, after shaking off and discarding any loose soil. No tubers were present at week 5 and, consequently, no potato geocaulosphere was harvested at that time. Soil samples were immediately frozen in liquid nitrogen and stored at $-80^{\circ} \mathrm{C}$.

Field trials. Two trials were conducted at the Potato Research Centre of Agriculture and Agri-Food Canada (Fredericton, NB, Canada) in an agricultural field (latitude $45^{\circ} 55^{\prime} 13.000^{\prime \prime} \mathrm{N}$, longitude $66^{\circ} 36^{\prime} 32.010^{\prime \prime} \mathrm{W}$, WMO ID: 71668) naturally containing common scab-causing pathogenic Streptomyces spp. The soil was a sandy loam
(58.7\% sand, $24.9 \%$ silt, and $16.4 \%$ clay) with a $\mathrm{pH}$ of 5.8 . The experiments were conducted from June to September 2012 and 2013 , as previously described (Arseneault et al. 2015). The field was divided into six (2013) or eight (2012) blocks, surrounded by guard rows, containing randomized treatment plots of 10 potato plants (Kennebec). Treatments included (i) control (no inoculation), (ii) single LBUM223 application, and (iii) biweekly applications with LBUM223 (2013 trial only). Inoculation with LBUM223 occurred at planting (single) and then also at 3, 5, 7, and 9 weeks for the biweekly application treatment. Control seed tubers received the same volume of water at planting. Due to regulation issues, the LBUM223 phzC-mutant strain was not used in the field trials. Destructive sampling of one randomly selected plant per plot occurred in the field at weeks 3, 5, 7, 9, and 11, for a total of six or eight plants per treatment per time. Plants were removed from the soil with their tubers intact and attached. Rhizosphere and geocaulosphere soils were collected as described in the growthchamber experiments. No tubers were present at weeks 3 and 5 and, consequently, no potato geocaulosphere was sampled at these times. All soil samples were immediately frozen in liquid nitrogen on site and subsequently stored at $-80^{\circ} \mathrm{C}$.

Extraction of DNA and RNA from soil. Rhizosphere and geocaulosphere soil samples were lyophilized prior to nucleic acid extractions. DNA was extracted using the UltraClean Soil DNA Isolation Kit (Mo Bio, Carlsbad, CA) following manufacturer's instructions, except for the vortex mixing step, which was substituted with the use of a FastPrep (QBiogene; MP Biomedicals, Santa Ana, $\mathrm{CA}$ ) at a speed of $5.5 \mathrm{~ms}^{-1}$ during $45 \mathrm{~s}$. Extracted DNA ( 25 to $\left.60 \mathrm{ng} / \mu \mathrm{l}\right)$ was diluted 1:10 prior to quantitative polymerase chain reaction (qPCR) analyses. The same soil samples were also submitted to RNA extraction using the protocol described by Bürgmann et al. (2003), with some modifications and two subsequent DNAse treatments, as previously described (Arseneault et al. 2013). RNA yield, measured using a NanoDrop spectrophotometer (NanoDrop Technologies, Wilmington, DE) was consistent between samples (between 150 and $400 \mathrm{ng} / \mu \mathrm{l})$. Prior to qPCR analyses, $11.55 \mu \mathrm{l}$ of nondiluted RNA was retro-transcribed using the 223 phzCfor primer (see below) $(200 \mathrm{nM})$ and the TaqMan Reverse Transcription Reagents Kit (Applied Biosystems, Foster City, CA) for a total reaction volume of $30 \mu \mathrm{l}$, which was used as is for qPCR analyses. Volumes rather than concentrations of nucleic acids were kept constant to maintain contaminants carried over from soil extractions at a similar concentration.

Development of primers and probe targeting $p h z C$. Novel primers and a TaqMan probe targeting $p h z C$ were developed using the Primer Express 3.0 software (Applied Biosystems), and were purchased from Integrated DNA Technologies (Coralville, IA) and Applied Biosystems, respectively. The primers and probe were designed using the available LBUM223 $\mathrm{phz} C$ gene sequence (GenBank accession number DQ788993). Because the target region used for designing the primers and the TaqMan probe in the $p h z C$ gene was not affected by the pKNOCK disruption in the isogenic $p h z C$ - insertional mutant (St-Onge et al. 2011), the primer/probe combination could be used to detect the populations of both the LBUM223 wild-type and $p h z C$ - mutant strains. BLASTn searches (version 2.2.30+; National Center for Biotechnology Information) (Altschul et al. 1997) performed on the primers and probe sequences revealed no significant matches to other genes and no exact match to $p h z C$ found in bacteria other than LBUM223. The primer/probe combination also did not yield any $p h z C$ amplicon when performing qPCR amplification on DNA extracted from nonsterile field soil (data not shown), confirming its specificity to LBUM223. Primer and probe sequences are as follows: 223phzCfor, 5'-ATA GAT GGA ATG CCG GTC ATG-3'; 223phzCrev, 5'-GCC CTC CTC CCT CTT TTG TTT-3'; and 223phzCprobe, 6FAM-5' -CGA CAA ACT CCA GTC AA-3'-MGBNFQ, generating an amplicon of $63 \mathrm{bp}$ with an amplification efficiency of $98 \%$.

qPCR and reverse-transcription qPCR analysis of phzC. Populations of LBUM223 (wild-type and isogenic phzC-mutant) were quantified using $\mathrm{qPCR}$, while the transcriptional activity of 
$p h z C$ was evaluated using reverse-transcription qPCR. Reactions $(20 \mu \mathrm{l})$ were prepared using the iTaq reagent, and consisted of $10 \mu \mathrm{l}$ of iTaq Supermix (Bio-Rad, Mississauga, ON, Canada), phzC primers and probe $(200 \mathrm{nM})$, and $4.8 \mu \mathrm{l}$ of cDNA or 1:10 diluted DNA (as previously described). Quantitative PCR assays were performed in triplicate and included no-template controls using a Bio-Rad CFX Connect (Bio-Rad). Each plate included a standard curve, determined by serial dilutions of a known number of plasmids containing $p h z C$, in order to obtain absolute gene copy numbers. The $p h z C$ gene is considered to be a single-copy gene in P. fluorescens (Mavrodi et al. 2010), and only one phz operon was detected in the sequenced LBUM223 genome (Roquigny et al. 2015); therefore, each copy of $p h z C$ detected was counted as one bacterial cell.

Physiological assays: biofilm formation and thaxtomin utilization. Characterization of some physiological traits of LBUM223 and its isogenic mutant potentially associated with rhizosphere or geocaulosphere colonization or survival was performed. The ability to form biofilms was assessed following the methods described by O'Toole and Kolter (1998) and modifications suggested by Selin et al. (2010). Briefly, both LBUM223 strains were grown at $25^{\circ} \mathrm{C}$ for $24 \mathrm{~h}$ in Luria-Bertani broth (BD). An optical density at $600 \mathrm{~nm}\left(\mathrm{OD}_{600}\right)$ reading was performed to ensure equal concentrations among all samples. Cultures were diluted 1:100 in M9 minimal salts medium with casamino acids (BD), supplemented with $0.2 \%$ glucose (wt/vol) and $1 \mathrm{mM} \mathrm{MgSO}_{4}$, of which $100 \mu \mathrm{l}$ was plated in each well of a 96-well polysterene cell culture plate (Nunclon Delta Surface; Thermo Scientific, Waltham, MA). Plates were incubated without shaking at $25^{\circ} \mathrm{C}$ for 24 and $48 \mathrm{~h}$, after which biofilm formation was assessed. The medium was removed and wells were washed once with water to remove all planktonic cells. Each well was stained with $125 \mu \mathrm{l}$ of crystal violet $(0.1 \%)$ for $10 \mathrm{~min}$, washed five times with water, air dried, and destained for 10 min with $200 \mu \mathrm{l}$ of $95 \%$ ethanol. The $\mathrm{OD}_{600}$ was measured by pipetting $125 \mu \mathrm{l}$ from each destained well into a new well and quantified using a plate reader (Varioskan; Thermo Scientific). For each culture and each time point, in addition to a control with medium only, eight replicate wells were used. The experiment was repeated a second time.

The capacity for utilizing thaxtomin as a nutrient source was also assessed following the protocol described by Doumbou et al. (1998). Minimal salts basal (MSB) medium was prepared as described (Doumbou et al. 1998), adding only a nitrogen source $\left(\left[\mathrm{NH}_{4}\right]_{2} \mathrm{SO}_{4}\right)$ when verifying thaxtomin as a carbon source (MSB-C), whereas only a carbon source (glycerol) was added when verifying the use of thaxtomin as a nitrogen source (MSB-N) by LBUM223 and its isogenic mutant. Modifications to the protocol were made: thaxtomin A was isolated as previously described (King and Lawrence 1996) and kindly provided by R. King (Agriculture and Agri-Food Canada). Thaxtomin A was added to the media at a concentration of $0.15 \mathrm{mM}$ for both nutrient assays, and bacterial growth was assessed by plating dilutions at $6,9,16,32$, and 64 days after inoculation. The experiment was done twice for both LBUM223 strains. Briefly, LBUM223 cultures were grown in TSB for 2 days in 50-ml tubes and quantified by spectrophotometry, as described above. All cultures were adjusted to $2 \times 10^{9} \mathrm{CFU} / \mathrm{ml}$. The cultures were centrifuged and washed twice with $0.85 \% \mathrm{NaCl}$ prior to resuspending cells in the initial culture volume in $0.85 \% \mathrm{NaCl}$. To inoculate $4 \mathrm{ml}$ of each media tested in duplicate, $10 \mu \mathrm{l}$ was used (bacterial concentration of $5 \times 10^{6} \mathrm{CFU} / \mathrm{ml}$ ), which included MSB-C and MSB-N containing thaxtomin, their respective negative controls (no thaxtomin), and a nutrient-rich TSB medium as a positive control. Growth was performed at $25^{\circ} \mathrm{C}$ (125 rpm) for 64 days, with 100 - $\mu$ l samples being collected at $6,9,16$, 32 , and 64 days, serially diluted in $0.85 \% \mathrm{NaCl}$, and plated in duplicate, from which counts were averaged to assess CFU/ml.

Statistical analyses. Three-factor (time, presence of $S$. scabies, and type of Pseudomonas sp. LBUM223 treatment for controlled conditions), two-factor (time and LBUM223 treatment for field conditions or time and LBUM223 strain for biofilm assays), or repeated-measures (thaxtomin utilization assay) analyses of variance were performed with subsequent Tukey-Kramer adjustment to determine significance $(P<0.05)$. Mixed models were used to include a random factor, which removes variation from repetitions used in controlled experiments and blocks used in field experiments. The interaction between factors was verified. Geocaulosphere and rhizosphere soils were analyzed separately. Population data (whether determined by qPCR or plate counts) required log transformations to achieve suitable homogeneity and normality. Gene expression data required rank transformations due to null values. Measures obtained from technical replicates originating from the same sample were averaged prior to statistical analysis (including qPCR triplicates). The SAS Statistical Analysis Software (v 9.3; SAS Institute, Cary, NC) was used for all analyses.

\section{RESULTS}

Rhizosphere and geocaulosphere soil population dynamics of LBUM223 in growth-chamber and field experiments. For the growth-chamber experiments (Fig. 1A and 1B), a single inoculation with LBUM223 or its $p h z C$ - mutant at planting enabled the establishment of up to $3 \times 10^{7}$ bacteria/g of rhizosphere soil at week 5 (Fig. 1B). However, populations significantly declined $(P<$ 0.03 ) over time, averaging approximately $9 \times 10^{6}$ bacteria/g of rhizosphere soil at week 10 and $1 \times 10^{6}$ bacteria/g of rhizosphere soil at week 15, regardless of the Pseudomonas treatment applied (LBUM223 wild type or $\mathrm{phzC}$ - mutant) or whether or not $S$. scabies was also present. In the geocaulosphere (Fig. 1A), populations of LBUM223 (wild type and $p h z C$ - mutant) at week 10 were similar to what was observed in the rhizosphere at the same time point; however, at week 15, results differed. An interaction between the time and $S$. scabies treatment factors showed that, at week 15 only, the presence of $S$. scabies maintained LBUM223 populations (both wild type and $p h z C$ - mutant) at the same level as those observed at week 10 whereas, in the absence of the pathogen, the populations of wild-type LBUM223 or its $p h z C$ - mutant significantly declined $(P<0.02)$. No qPCR amplification was detected in samples originating from pots that were not inoculated with LBUM223 (wild type or $p h z C-$ mutant) (data not shown).

In the first field trial (2012), populations of LBUM223 were quantified at different time points following a single inoculation at planting. Because the rhizospheric populations were significantly reduced during the course of the experiment (Supplementary Fig. S1), a biweekly application treatment was added to the 2013 replication field trial to determine whether reapplying LBUM223 could affect population levels. The same general trends were obtained in the 2012 and 2013 field experiments; therefore, the results of the 2013 experiment only are shown in Figure 2, allowing the comparison of a single application with LBUM223 to the biweekly application treatment. In the rhizosphere (Fig. 2B) at week 3 , both the single and biweekly application treatments had received only one inoculation at planting, and populations reached approximately $3 \times 10^{7}$ bacteria/g of soil, which was similar to what was observed under controlled growth-chamber conditions. In subsequent weeks, there was a clear decline (to $2 \times 10^{5}$ bacteria/g of rhizosphere) in LBUM223 populations for plants having received a single application. However, with biweekly applications, the populations of LBUM223 remained relatively stable, with the exception of week 9, where populations were slightly lower. At each time point, except week 3, populations of LBUM223 in the biweekly application treatment were significantly higher than in the single application treatment. Statistical differences are presented as a result of multiple comparisons of all treatments at all times, because an interaction occurred between the time and treatment factors $(P<$ 0.0001) (Fig. 2B). A similar trend was also observed in the geocaulosphere (Fig. 2A) $(P=0.0001)$. At weeks 9 and 11, LBUM223 populations in the biweekly application treatment were almost 10 times higher than in the single application treatment. 
Quantification of $p h z C$ gene transcripts in the rhizosphere and geocaulosphere soils of potato grown in growth-chamber and field experiments. In growth-chamber experiments (Fig. 3), an effect due to the inoculation treatments was only found in the geocaulosphere, where the number of $p h z C$ transcripts produced by LBUM223 was significantly greater when $S$. scabies was present compared with when only LBUM223 was applied, where no transcripts were detected (Fig. 3A) $(P<0.0001)$. Time did not affect transcript abundance in the geocaulosphere, which varied between $10^{1}$ and $10^{3}$ transcripts/g of soil when detected. In the rhizosphere (Fig. 3B), there was no significant effect due to treatments; however, there were differences observed in time. At week 5, transcripts were readily detectable and significantly more abundant (averaging approximately $5 \times 10^{1}$ transcripts/g of soil) compared with weeks 10 and 15 (fewer than 10 transcripts/g of soil) $(P<0.003$, significant difference in time not shown) (Fig. 3B). Transcripts were not detected at week 10 for the LBUM223 treatment and at week 15 for the LBUM $223+$ S. scabies treatment.
As expected, $p h z C$ transcripts were not detected in soil inoculated with LBUM223 phzC-.

In field experiments (Fig. 4), biweekly applications with LBUM223 led to significantly more $p h z C$ transcripts detected in both the geocaulosphere (Fig. 4A) $(P<0.02)$ and rhizosphere (Fig. 4B) $(P<0.0001)$ compared with the single application treatment. However, expression generally declined over time. In the geocaulosphere, the number of transcripts at week 11 was significantly lower $\left(1 \times 10^{3}\right)$ than at weeks 7 and $9\left(1 \times 10^{4}\right)(P<0.006$, significant difference in time not shown) (Fig. 4A). In the rhizosphere, the abundance of $p h z C$ transcripts was significantly higher at week 5 $\left(2 \times 10^{5}\right)$ compared with weeks 9 and $11\left(2 \times 10^{3}\right)(P<0.02$, significant difference in time not shown) (Fig. $4 \mathrm{~B}$ ). No $\mathrm{phz} C$ transcripts were detected from the control (noninoculated) rhizosphere and geocaulosphere soil samples.

In both the growth-chamber and field experiments, although the total number of $p h z C$ transcripts detected per gram of soil varied according to treatments, it was not significantly different when
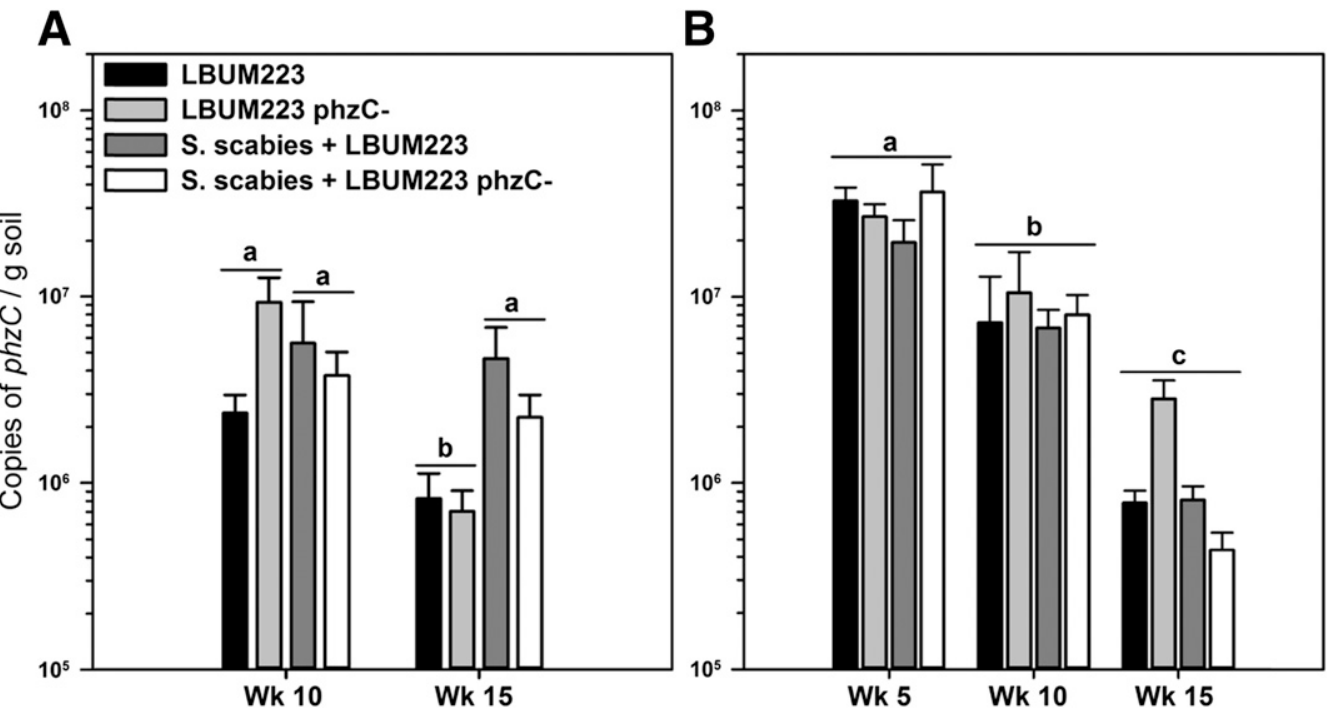

Fig. 1. Soil populations of wild-type Pseudomonas fluorescens LBUM223 or its $p h z C$ - isogenic mutant, in the A, geocaulosphere and $\mathbf{B}$, rhizosphere soils in growthchamber trials. Values are presented as mean \pm standard error of the mean $(n=6)$. A three-factor analysis of variance determined that an interaction occurred between time and Streptomyces scabies treatment factors in the geocaulosphere only. Significant differences $(P<0.02)$ are presented using lowercase letters.

\section{A}

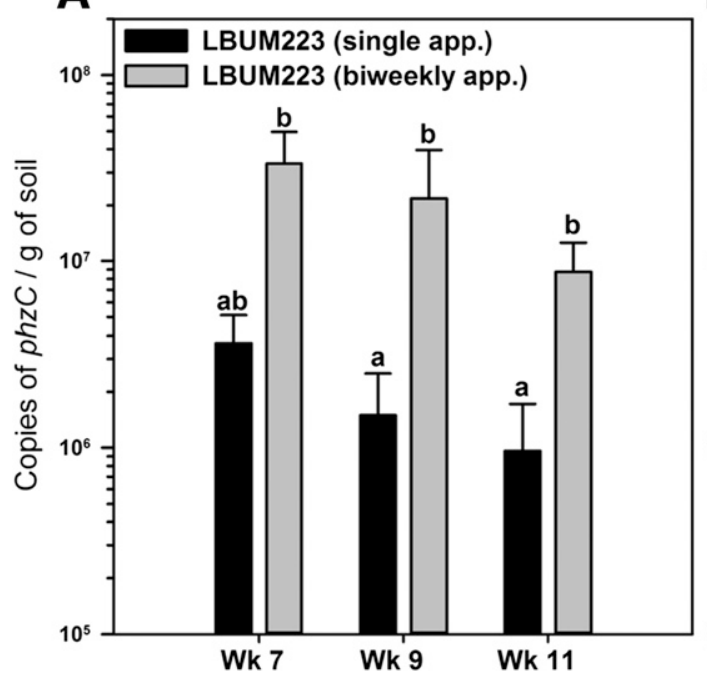

B

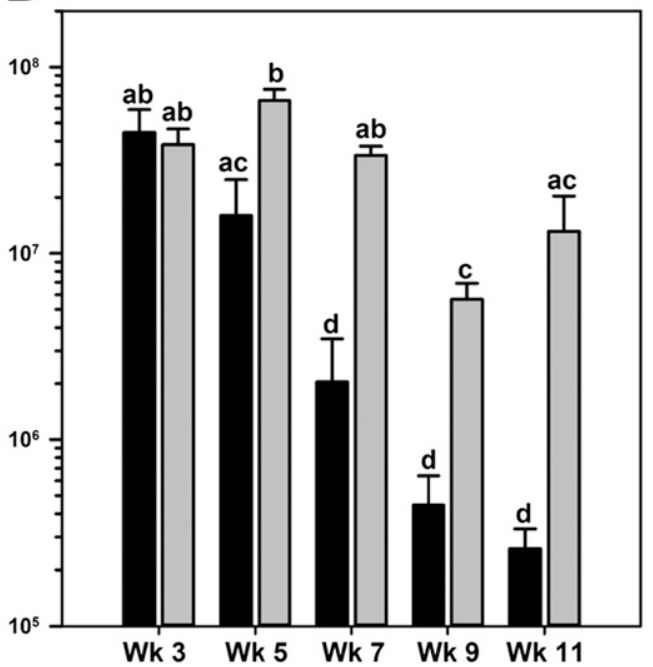

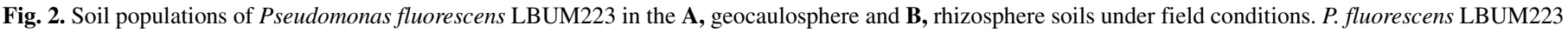

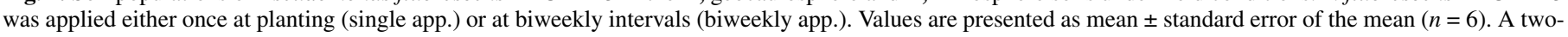

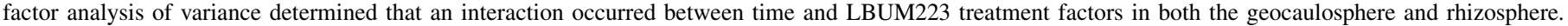
Significant differences $(P<0.0001)$ are presented using lowercase letters. 
expressed by numbers of $p h z C$ transcripts per bacteria (data not shown). Expression of $p h z C$ was approximately 500 times higher in field experiments (averaging $5 \times 10^{-3}$ transcripts/bacteria) than in growth-chamber experiments (averaging $1 \times 10^{-5}$ transcripts/ bacteria) (data not shown).

Characterization of LBUM223 biofilm and thaxtomin utilization. Biofilm assays showed that LBUM223 and its isogenic phzC-mutant were equally able to form biofilms after 24 and $48 \mathrm{~h}$ (Supplementary Fig. S2). The bacteria aggregated in the form of a ring on the well surface, at the air-liquid interface. For the thaxtomin utilization assay, a global statistical analysis at all time points combined (Supplementary Fig. S3A to C) showed that LBUM223 and its isogenic $p h z C$ - mutant were equally able to utilize thaxtomin as a source of carbon but not nitrogen. Also of interest was the ability of LBUM223 to maintain stable and viable cells at the same concentration inoculated $\left(10^{6} \mathrm{CFU} / \mathrm{ml}\right)$ in carbon- or nitrogen-depleted minimal media for at least 2 months.

\section{DISCUSSION}

This study aimed at characterizing the population dynamics of LBUM223, as well as the expression of the PCA biosynthetic gene $p h z C$ in the geocaulosphere and rhizosphere of potato, to better understand the implication of LBUM233 in the biocontrol of common scab. In growth-chamber experiments, it was possible to study the effect of the presence or absence of S. scabies on the population dynamics of LBUM223. Results showed that the presence of $S$. scabies prevented a time-dependent reduction in
A

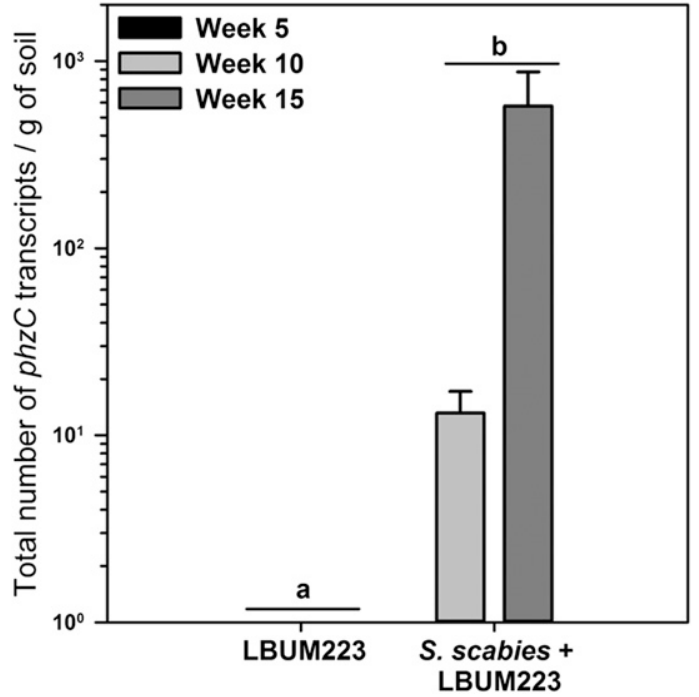

B

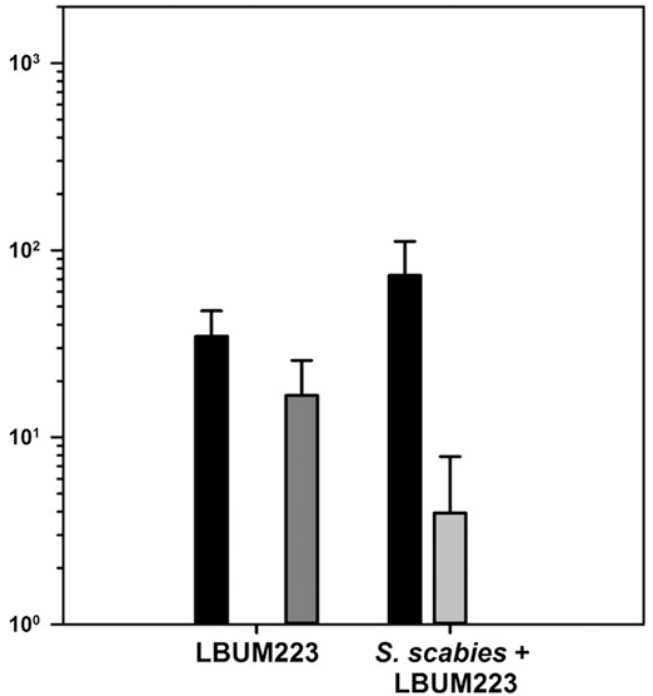

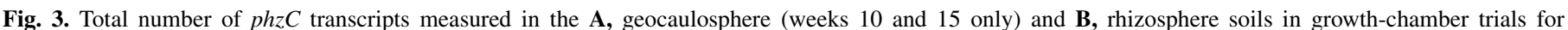

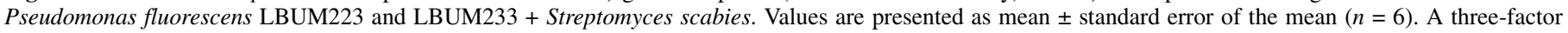

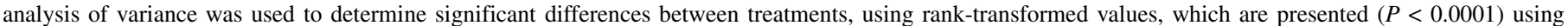
lowercase letters.
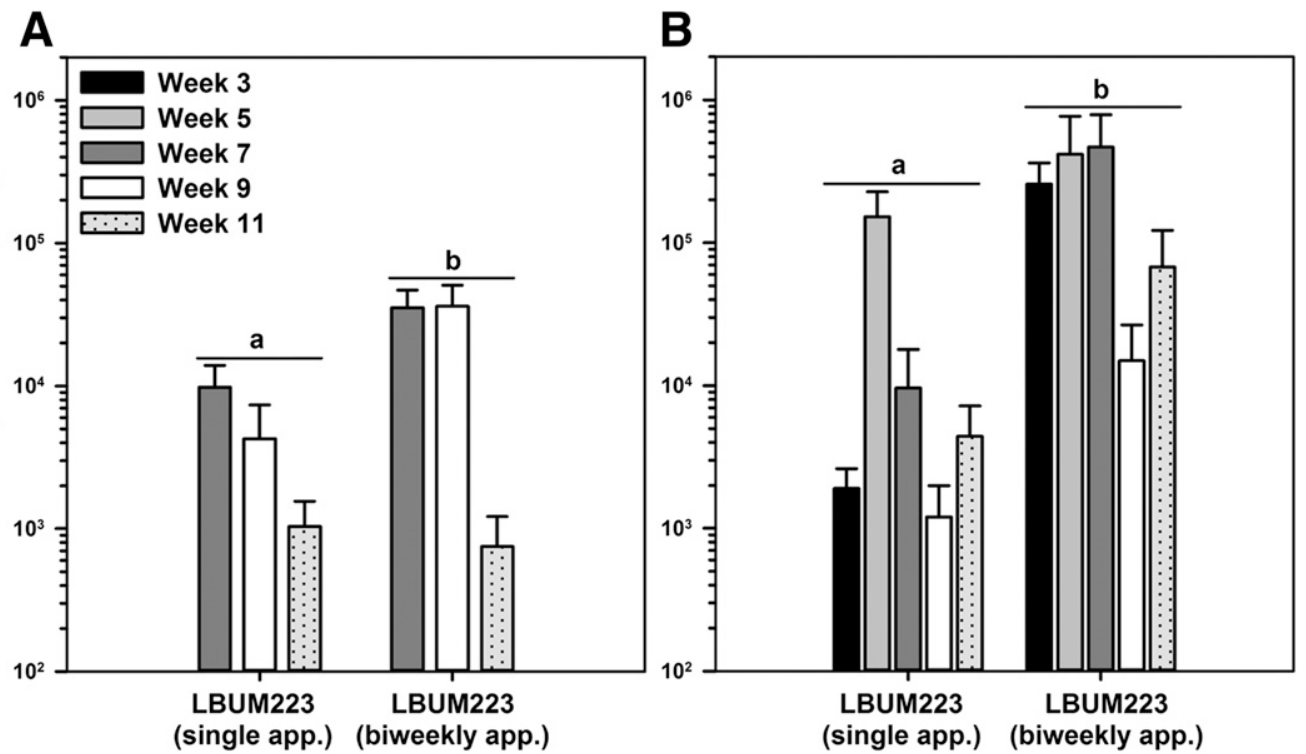

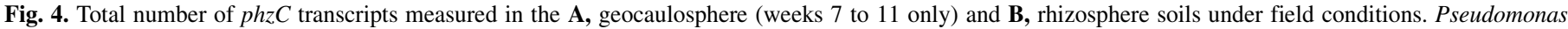

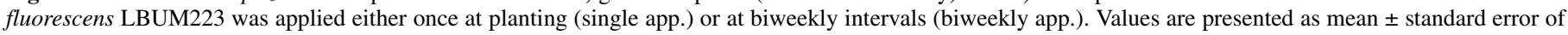

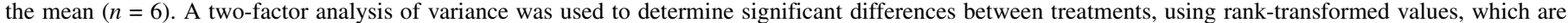
presented $(P<0.02)$ using lowercase letters. 
populations of LBUM223 in the geocaulosphere, the site of action of the pathogen. A previous study has also reported on two Pseudomonas strains which maintained or increased their soil populations up to 1 month after exposure to two fungal wheat pathogens (causing take-all and Rhizoctonia root rot); however, the mechanisms involved were not described (Mazzola and Cook 1991). Interestingly, previous work performed in our laboratory has shown that a reduction in virulence gene expression (encoding thaxtomin A production) in S. scabies, which was linked to disease control, was also only found in the geocaulosphere following wildtype LBUM223 inoculation (Arseneault et al. 2013). This suggests that the LBUM223-S. scabies interaction leading to biocontrol occurs mainly in this specific soil environment. Production of thaxtomin $\mathrm{A}$ is also known to be higher in the geocaulosphere than in the rhizosphere in response to rapidly growing tuber tissues and also due to the pathogen's growth in developing lesions (Loria et al. 2008). Therefore, the presence of thaxtomin A could be a determining factor in the increased LBUM223 populations observed when $S$. scabies is present, even if this production is likely reduced but not eliminated when exposed to LBUM223 (Arseneault et al. 2013). Some bacterial strains of nonpathogenic Streptomyces and Ralstonia spp. have been shown capable of utilizing thaxtomin A as a carbon and nitrogen source (Doumbou et al. 1998); therefore, one possible explanation is that LBUM223 is able to utilize it as a nutrient source. The thaxtomin utilization assays preformed in this study show that both wild-type and $p h z C$ - strains of LBUM223 are able to utilize thaxtomin as a carbon source, even at low concentrations, which possibly explains why both strains are able to sustain growth in the presence of $S$. scabies. However, because only wild-type LBUM223 is able to reduce disease symptoms, this suggests that the utilization of thaxtomin is not likely to be sufficient to reduce virulence as a means of detoxification. Another possible explanation as to why both wild-type and $\mathrm{phzC}$-strains of LBUM223 can sustain their populations in the geocaulosphere only could be the presence of infected tubers, even though lesions show different degrees of severity when treated with LBUM223 or its mutant. Scab symptoms caused by $S$. scabies could facilitate the provision of additional nutrients to LBUM223 (wild type or mutant) by compromising the tubers' protective skin barrier (to be confirmed).

The population dynamics of wild-type LBUM223 as well as its isogenic $p h z C$ - mutant were similar, with both strains being equally able to establish themselves in the geocaulosphere and rhizosphere soils at all times during controlled growth-chamber experiments. Their similar colonization patterns can be explained by their equivalent ability to form biofilms, a critical structure for establishing colonies in soil and on plant roots (Ramey et al. 2004). The fact that the absence of PCA production in the $p h z C$ - mutant had no effect on biofilm formation was surprising because phenazine production is thought to contribute to the formation of biofilms in many Pseudomonas spp. (Maddula et al. 2006; Mavrodi et al. 2013; Wang et al. 2011). However, because nonphenazine-producing Pseudomonas spp. have also been reported capable of forming biofilms, including $P$. fluorescens strains such as Pf-01, WCS365, and SBW25 (Loper et al. 2012; Ude et al. 2006), it is not the first mention of a non-PCA-producing $P$. fluorescens being able to form biofilms.

For field experiments, where only wild-type LBUM223 was used, results showed that biweekly inoculations with LBUM223 maintained populations at approximately $10^{7}$ bacteria/g of soil in both the geocaulosphere and rhizosphere. A single inoculation with LBUM223 at planting resulted in a time-dependent reduction in LBUM223 populations in the rhizosphere to approximately $10^{5}$ bacteria/g after 9 weeks, a more drastic reduction in populations than the one occurring under controlled conditions. The presence of numerous other microorganisms competing with LBUM223 in field soil compared with the sterilized field soil used under controlled conditions could explain this result. In addition, other environmental variations such as soil water content and temperature variations found under natural field conditions could also account for the greater rate of LBUM223 population decline in the field compared with controlled conditions, because these have been found to affect the root colonization capacity of several Pseudomonas spp. (Loper et al. 1985; Mavrodi et al. 2012). Decline in soil populations of inoculated Pseudomonas spp. has often been reported to occur. Kloepper et al. (1980) also found a significant reduction in potato growth-promoting Pseudomonas spp. populations in field rhizosphere samples during a growing season by as much as $10^{2}$, and populations of Pseudomonas spp. strains CHA0, F113, and Pf153 in the rhizosphere of maize grown in pots containing field soil followed a similar trend, decreasing by approximately two logs from week 1 to week 5 (Von Felten et al. 2010). The results obtained in this study suggest that a sufficient establishment of LBUM223 populations is critical for biocontrol under field conditions, because only the biweekly application treatment was successful in significantly reducing common scab symptoms (Arseneault et al. 2015).

In addition to characterizing LBUM223 population's dynamics, the transcriptional activity of $p h z C$ was quantified in controlled and field conditions. As expected, $p h z C$ transcripts were only detected in treatments having received wild-type LBUM223, and were not detected in the LBUM223 phzC-inoculated or control treatments. Under controlled conditions, the presence of $S$. scabies in the geocaulosphere resulted in significantly more $\mathrm{phz} C$ transcripts being detected than in the absence of the pathogen while, in the field study, biweekly applications of LBUM223 also led to a greater abundance of $p h z C$ transcripts compared with the single inoculation treatment. Although the presence of $S$. scabies did not increase the number of $p h z C$ transcripts detected per bacteria, the increased population size of LBUM223 between treatments with and without $S$. scabies and also between treatments with single and biweekly inoculation with LBUM223 resulted in a higher number of $p h z C$ transcripts being detected. A study by Mavrodi et al. (2012) has shown that a direct relationship exists between the size of indigenous populations of phenazine-producing Pseudomonas spp. and the quantity of PCA extracted from field soil. A direct chemical measurement of PCA production, however, was not performed in our study because less than $5 \mathrm{~g}$ of soil could be retrieved from geocaulosphere soil samples, which is well below the $15 \mathrm{~g}$ of root and adhering soil required for efficient PCA quantification, as described in the protocol developed by Mavrodi et al. (2012). However, we can speculate that the increase in PCA-producing LBUM223 populations and, consequently, the increased $p h z C$ transcript numbers being produced led to greater amounts of PCA in soil and better biocontrol.

Interestingly, the amounts of $p h z C$ transcripts detected in field soil samples were much greater than in growth-chamber soil samples (between 10 and $10^{3}$ times higher), even though LBUM223 soil populations were fairly similar and the preparation of the inocula and the concentration applied were identical in both cases. Factors contributing to this difference are unknown; however, one possibility is that the presence of a wide variety of indigenous microorganisms in the field, along with their production of various metabolites, may account for this. The expression of the phz operon is regulated by homoserine lactone (HSL)-mediated quorum-sensing (Wood and Pierson 1996), a diffusible signal produced by many gram-negative bacterial species, including Pseudomonads (Fuqua et al. 2001; Latifi et al. 1995; Pierson et al. 1998). Therefore, the presence of other HSLproducing bacteria in the field may have contributed to enhancing $\mathrm{phz} C$ expression. Also, environmental factors, including $\mathrm{pH}$, temperature, and nutrient availability (Slininger and Shea-Wilbur 1995), as well as soil texture, microelement, and inorganic nitrogen concentrations (Ownley et al. 2003), which differ between controlled and field conditions, could perhaps explain the increased transcriptional activity of the PCA biosynthetic operon detected in the field. 
Taken together, our findings suggest that LBUM223 must colonize the geocaulosphere of potato at high levels in order to achieve biocontrol of common scab. Such high population levels (approximately $10^{7}$ bacteria/g of soil) lead to greater $p h z C$ transcript accumulation, and likely to a higher concentration of PCA, which can be associated with a reduction in common scab symptoms. The presence of $S$. scabies appears to be a factor that contributes to increasing LBUM223 populations and $p h z C$ transcript accumulation in the geocaulosphere. Under field conditions, reapplying LBUM223 during the growing season appears crucial to prevent soil population decline occurring over time. The contribution of this work to better understanding the interactions occurring between LBUM223 and S. scabies under controlled and natural field conditions has provided knowledge that will most likely be useful in further developing $P$. fluorescens LBUM223 as an efficient commercial biocontrol agent.

\section{ACKNOWLEDGMENTS}

This study was supported by Natural Sciences and Engineering Research Council (NSERC) and New Brunswick Innovation Foundation grants to M. Filion. A doctoral Alexander Graham Bell Canada Graduate Scholarship (NSERC) was awarded to T. Arseneault. We thank C. Lanteigne, A. Novinscak, C. Morrison (Université de Moncton, Moncton, NB, Canada), and S. Whitney (Agriculture and Agri-Food Canada, Fredericton, NB, Canada) for their much appreciated technical assistance; R. King (Agriculture and AgriFood Canada, Fredericton) for providing thaxtomin A; and G. Moreau (Université de Moncton) for sharing suggestions regarding statistical analyses.

\section{LITERATURE CITED}

Altschul, S. F., Madden, T. L., Schäffer, A. A., Zhang, J., Zhang, Z., Miller, W., and Lipman, D. J. 1997. Gapped BLAST and PSI-BLAST: A new generation of protein database search programs. Nucleic Acids Res. 25: 3389-3402.

Arseneault, T., Goyer, C., and Filion, M. 2013. Phenazine production by Pseudomonas sp. LBUM223 contributes to the biological control of potato common scab. Phytopathology 103:995-1000.

Arseneault, T., Goyer, C., and Filion, M. 2015. Pseudomonas fluorescens LBUM223 increases potato yield and reduces common scab symptoms in the field. Phytopathology 105:1311-1317.

Bürgmann, H., Widmer, F., Sigler, W. V., and Zeyer, J. 2003. mRNA extraction and reverse transcription-PCR protocol for detection of nifH gene expression by Azotobacter vinelandii in soil. Appl. Environ. Microbiol. 69: 1928-1935.

Chin-A-Woeng, T. F. C., Bloemberg, G. V., and Lugtenberg, B. J. J. 2003. Phenazines and their role in biocontrol by Pseudomonas bacteria. New Phytol. 157:503-523.

D'aes, J., Hua, G. K. H., De Maeyer, K., Pannecoucque, J., Forrez, I., Ongena, M., Dietrich, L. E. P., Thomashow, L. S., Mavrodi, D. V., and Höfte, M. 2011. Biological control of Rhizoctonia root rot on bean by phenazine- and cyclic lipopeptide-producing Pseudomonas CMR12a. Phytopathology 101: 996-1004.

Doumbou, C., Akimov, V., and Beaulieu, C. 1998. Selection and characterization of microorganisms utilizing thaxtomin A, a phytotoxin produced by Streptomyces scabies. Appl. Environ. Microbiol. 64:4313-4316.

Fuqua, C., Parsek, M. R., and Greenberg, E. P. 2001. Regulation of gene expression by cell-to-cell communication: Acyl-homoserine lactone quorum sensing. Annu. Rev. Genet. 35:439-468.

Haas, D., and Défago, G. 2005. Biological control of soil-borne pathogens by fluorescent pseudomonads. Nat. Rev. Microbiol. 3:307-319.

King, R. R., and Lawrence, C. H. 1996. Characterization of new thaxtomin A analogues generated in vitro by Streptomyces scabies. J. Agric. Food Chem. 44:1108-1110.

Kloepper, J. W., Schroth, M. N., and Miller, T. D. 1980. Effects of rhizosphere colonization by plant growth-promoting rhizobacteria on potato plant development and yield. Phytopathology 70:1078-1082.

Lambert, D., and Loria, R. 1989. Streptomyces scabies sp. nov., nom. rev. Int. J. Syst. Bacteriol. 39:387-392.

Latifi, A., Winson, M. K., Foglino, M., Bycroft, B. W., Stewart, G. S., Lazdunski, A., and Williams, P. 1995. Multiple homologues of LuxR and LuxI control expression of virulence determinants and secondary metabolites through quorum sensing in Pseudomonas aeruginosa PAO1. Mol. Microbiol. 17:333-343.
Loper, J. E., Haack, C., and Schroth, M. N. 1985. Population dynamics of soil Pseudomonads in the rhizosphere of potato (Solanum tuberosum L. ). Appl. Environ. Microbiol. 49:416-422.

Loper, J. E., Hassan, K. A., Mavrodi, D. V., Davis, E. W., II, Lim, C. K., Shaffer, B. T., Elbourne, L. D. H., Stockwell, V. O., Hartney, S. L., Breakwell, K., Henkels, M. D., Tetu, S. G., Rangel, L. I., Kidarsa, T. A., Wilson, N. L., van de Mortel, J. E., Song, C., Blumhagen, R., Radune, D., Hostetler, J. B., Brinkac, L. M., Durkin, A. S., Kluepfel, D. A., Wechter, W. P., Anderson, A. J., Kim, Y. C., Pierson, L. S., III, Pierson, E. A., Lindow, S. E., Kobayashi, D. Y., Raaijmakers, J. M., Weller, D. M., Thomashow, L. S., Allen, A. E., and Paulsen, I. T. 2012. Comparative genomics of plant-associated Pseudomonas spp.: Insights into diversity and inheritance of traits involved in multitrophic interactions. PLoS Genet. 8: e1002784.

Loria, R., Bignell, D. R. D., Moll, S., Huguet-Tapia, J. C., Joshi, M. V., Johnson, E. G., Seipke, R. F., and Gibson, D. M. 2008. Thaxtomin biosynthesis: The path to plant pathogenicity in the genus Streptomyces. Antonie Leeuwenhoek 94:3-10.

Maddula, V. S. R. K., Zhang, Z., Pierson, E. A., and Pierson, L. S., III. 2006. Quorum sensing and phenazines are involved in biofilm formation by Pseudomonas chlororaphis (aureofaciens) strain 30-84. Microb. Ecol. 52: 289-301.

Mavrodi, D. V., Ksenzenko, V. N., Bonsall, R. F., Cook, R. J., Boronin, A. M., and Thomashow, L. S. 1998. A seven-gene locus for synthesis of phenazine1-carboxylic acid by Pseudomonas fluorescens 2-79. J. Bacteriol. 180: 2541-2548.

Mavrodi, D. V., Mavrodi, O. V., Parejko, J. A., Bonsall, R. F., Kwak, Y.-S., Paulitz, T. C., Thomashow, L. S., and Weller, D. M. 2012. Accumulation of the antibiotic phenazine-1-carboxylic acid in the rhizosphere of dryland cereals. Appl. Environ. Microbiol. 78:804-812.

Mavrodi, D. V., Parejko, J. A., Mavrodi, O. V., Kwak, Y.-S., Weller, D. M., Blankenfeldt, W., and Thomashow, L. S. 2013. Recent insights into the diversity, frequency and ecological roles of phenazines in fluorescent Pseudomonas spp. Environ. Microbiol. 15:675-686.

Mavrodi, D. V., Peever, T. L., Mavrodi, O. V., Parejko, J. A., Raaijmakers, J. M., Lemanceau, P., Mazurier, S., Heide, L., Blankenfeldt, W., Weller, D. M., and Thomashow, L. S. 2010. Diversity and evolution of the phenazine biosynthesis pathway. Appl. Environ. Microbiol. 76:866-879.

Mazurier, S., Corberand, T., Lemanceau, P., and Raaijmakers, J. M. 2009. Phenazine antibiotics produced by fluorescent pseudomonads contribute to natural soil suppressiveness to Fusarium wilt. ISME J. 3:977-991.

Mazzola, M., and Cook, R. 1991. Effects of fungal root pathogens on the population dynamics of biocontrol strains of fluorescent pseudomonads in the wheat rhizosphere. Appl. Environ. Microbiol. 57:2171-2178.

O'Toole, G., and Kolter, R. 1998. Initiation of biofilm formation in Pseudomonas fluorescens WCS365 proceeds via multiple, convergent signalling pathways: A genetic analysis. Mol. Microbiol. 28:449-461.

Ownley, B. H., Duffy, B. K., and Weller, D. M. 2003. Identification and manipulation of soil properties to improve the biological control performance of phenazine-producing Pseudomonas fluorescens. Appl. Environ. Microbiol. 69:3333-3343.

Pierson, L. S., III, and Pierson, E. 2010. Metabolism and function of phenazines in bacteria: Impacts on the behavior of bacteria in the environment and biotechnological processes. Appl. Microbiol. Biotechnol. 86:1659-1670.

Pierson, L. S., III, Wood, D. W., Pierson, E. A., and Chancey, S. T. 1998. $\mathrm{N}$-acyl-homoserine lactone-mediated gene regulation in biological control by fluorescent pseudomonads: Current knowledge and future work. Eur. J. Plant Pathol. 104:1-9.

Price-Whelan, A., Dietrich, L. E. P., and Newman, D. K. 2006. Rethinking "secondary" metabolism: Physiological roles for phenazine antibiotics. Nat. Chem. Biol. 2:71-78.

Raaijmakers, J. M., Vlami, M., and de Souza, J. T. 2002. Antibiotic production by bacterial biocontrol agents. Antonie Leeuwenhoek 81:537-547.

Raio, A., Puopolo, G., Cimmino, A., Danti, R., Della Rocca, G., and Evidente, A. 2011. Biocontrol of cypress canker by the phenazine producer Pseudomonas chlororaphis subsp. aureofaciens strain M71. Biol. Control 58:133-138.

Ramey, B. E., Koutsoudis, M., von Bodman, S. B., and Fuqua, C. 2004. Biofilm formation in plant-microbe associations. Curr. Opin. Microbiol. 7: 602-609.

Roquigny, R., Arseneault, T., Gadkar, V. J., Novinscak, A., Joly, D. L., and Filion, M. 2015. Complete genome sequence of the biocontrol strain Pseudomonas fluorescens LBUM223. Genome Announc. 3:e00443-15.

Selin, C., Habibian, R., Poritsanos, N., Athukorala, S. N. P., Fernando, D., and de Kievit, T. R. 2010. Phenazines are not essential for Pseudomonas chlororaphis PA23 biocontrol of Sclerotinia sclerotiorum, but do play a role in biofilm formation. FEMS Microbiol. Ecol. 71:73-83.

Slininger, P., and Jackson, M. 1992. Nutritional factors regulating growth and accumulation of phenazine 1-carboxylic acid by Pseudomonas fluorescens 2-79. Appl. Microbiol. Biotechnol. 37:388-392. 
Slininger, $\mathrm{P}$, and Shea-Wilbur, M. 1995. Liquid-culture $\mathrm{pH}$, temperature, and carbon (not nitrogen) source regulate phenazine productivity of the take-all biocontrol agent Pseudomonas fluorescens 2-79. Appl. Microbiol. Biotechnol. 43:794-800.

St-Onge, R., Gadkar, V. J., Arseneault, T., Goyer, C., and Filion, M. 2011. The ability of Pseudomonas sp. LBUM223 to produce phenazine-1carboxylic acid affects the growth of Streptomyces scabies, the expression of thaxtomin biosynthesis genes and the biological control potential against common scab of potato. FEMS Microbiol. Ecol. 75: 173-183.

St-Onge, R., Goyer, C., Coffin, R., and Filion, M. 2008. Genetic diversity of Streptomyces spp. causing common scab of potato in eastern Canada. Syst. Appl. Microbiol. 31:474-484.

Thomashow, L. S., Weller, D. M., Bonsall, R. F., and Pierson, L. S., III. 1990. Production of the antibiotic phenazine-1-carboxylic acid by fluorescent Pseudomonas species in the rhizosphere of wheat. Appl. Environ. Microbiol. 56:908-912.

Timms-Wilson, T. M., Ellis, R. J., Renwick, A., Rhodes, D. J., Mavrodi, D. V., Weller, D. M., Thomashow, L. S., and Bailey, M. J. 2000. Chromosomal insertion of phenazine-1-carboxylic acid biosynthetic pathway enhances efficacy of damping-off disease control by Pseudomonas fluorescens. Mol. Plant-Microbe Interact. 13:1293-1300.
Ude, S., Arnold, D. L., Moon, C. D., Timms-Wilson, T., and Spiers, A. J. 2006. Biofilm formation and cellulose expression among diverse environmental Pseudomonas isolates. Environ. Microbiol. 8:1997-2011.

Von Felten, A., Défago, G., and Maurhofer, M. 2010. Quantification of Pseudomonas fluorescens strains F113, CHA0 and Pf153 in the rhizosphere of maize by strain-specific real-time PCR unaffected by the variability of DNA extraction efficiency. J. Microbiol. Methods 81:108-115.

Wang, Y., Wilks, J. C., Danhorn, T., Ramos, I., Croal, L., and Newman, D. K. 2011. Phenazine-1-carboxylic acid promotes bacterial biofilm development via ferrous iron acquisition. J. Bacteriol. 193:3606-3617.

Weller, D. M. 2007. Pseudomonas biocontrol agents of soilborne pathogens: Looking back over 30 years. Phytopathology 97:250-256.

Weller, D. M., Raaijmakers, J. M., McSpadden Gardener, B. B., and Thomashow, L. S. 2002. Microbial populations responsible for specific soil suppressiveness to plant pathogens. Annu. Rev. Phytopathol. 40:309-348.

Wood, D., and Pierson, L. S., III. 1996. The phzI gene of Pseudomonas aureofaciens 30-84 is responsible for the production of a diffusible signal required for phenazine antibiotic production. Gene 168:49-53.

Yang, M.-M., Mavrodi, D. V., Mavrodi, O. V., Bonsall, R. F., Parejko, J. A., Paulitz, T. C., Thomashow, L. S., Yang, H.-T., Weller, D. M., and Guo, J.-H. 2011. Biological control of take-all by fluorescent Pseudomonas spp. from Chinese wheat fields. Phytopathology 101:1481-1491. 